\title{
Transcriptional regulation of HERV-E expression in clear cell renal carcinoma
}

\author{
Elena Cherkasova, Michael I Lerman and Richard Childs*
}

\author{
Address: NHLBI, NIH, Bethesda, MD, USA
}

* Corresponding author

\author{
from Frontiers of Retrovirology: Complex retroviruses, retroelements and their hosts \\ Montpellier, France. 21-23 September 2009 \\ Published: 24 September 2009 \\ Retrovirology 2009, 6(Suppl 2):P23 doi:10.1 186/1742-4690-6-S2-P23
}

This abstract is available from: http://www.retrovirology.com/content/6/S2/P23

(c) 2009 Cherkasova et al; licensee BioMed Central Ltd.

\section{Background}

We recently discovered two novel transcripts (CT-RCC-8 and CT-RCC-9) from HERV-E provirus located on chromosome $6 \mathrm{q}$ expressed at variable levels in $>50 \%$ of renal cell carcinoma (RCC) samples but not in normal tissues or other tumors. We also identified a peptide antigen encoded by both of these HERV-E transcripts that was recognized by RCC-reactive T-cells isolated from a patient with metastatic RCC who had dramatic regression of tumor following a non-myeloablative allogeneic stem cell transplant [1].

\section{Results}

Here, we show that HERV-E expression occurs only in clear cell variants of RCC (ccRCC) that demonstrate loss of normal Von Hippel-Lindau (VHL) protein. VHL transgenes introduced into VHL deficient ccRCC cell lines suppressed HERV-E expression implying that VHL controls provirus activity. Loss of functional pVHL has previously been shown to increase expression of the hypoxia-inducible factors HIF- $1 \alpha$ and HIF- $2 \alpha$. We found a strong correlation between the transcriptional activity of HIF- $2 \alpha$ and HERV-E provirus activation. We also found that expression of the HERV-E in cCRCC cell lines correlated with the demethylation of the 5'LTR while normal tissues and cCRCC cell lines without HERV-E expression showed strong CpG methylation in this region. Once activated, HIF-2 $\alpha$ binds DNA at the consensus HRE (core HRE sequence is CGTG) in the target gene promoters, and a HRE motif was identified in 5'LTR of the HERV-E. It is therefore possible that methylation of 5'LTR CpGs including the HRE CPG can explain silencing of HERV-E even in the presence of active transcriptional factors. The mechanism protecting the HERV-E promoter from methylation in VHL defective ccRCC lines leading to expression of proviral genes remains under investigation.

\section{Conclusion}

These findings provide insight into mechanisms regulating HERV activation in human malignancies. The contribution of HERV-E to kidney tumorigenesis warrants further elucidation.

\section{References}

I. Takahashi Y, Harashima N, Kajigaya S, Yokoyama H, Cherkasova E, McCoy JP, Hanada K, Mena O, Kurlander R, Abdul T, Srinivasan R, Lundqvist A, Malinzak E, Geller N, Lerman MI, Childs R: Regression of kidney cancer following allogeneic stem-cell transplantation associated with T-cells recognizing a HERV-E antigen. J Clin Invest 2008, I I 8:1099-1 I09. 